\title{
PiSZ alpha-1 antitrypsin deficiency (AATD): pulmonary phenotype and prognosis relative to PiZZ AATD and PiMM COPD
}

\author{
C E Green, ${ }^{1}$ S Vayalapra, ${ }^{1}$ J A Hampson, ${ }^{1}$ D Mukherjee, ${ }^{2}$ R A Stockley, ${ }^{1}$ \\ A M Turner ${ }^{1,3}$
}

- Additional material is published online only. To view please visit the journal online (http://dx.doi.org/10.1136/ thoraxjnl-2015-206906).

${ }^{1}$ Centre for Translational Inflammation Research, University of Birmingham, Birmingham, UK

${ }^{2}$ Imperial College, London, UK ${ }^{3}$ Heart of England NHS Foundation Trust, Birmingham, UK

\section{Correspondence to} Dr AM Turner, Centre for Translational Inflammation Research, University of Birmingham, Birmingham, B15 2WB, UK:

a.m.wood@bham.ac.uk

Received 9 February 2015 Revised 9 June 2015 Accepted 15 June 2015 Published Online First 3 July 2015

\section{CrossMark}

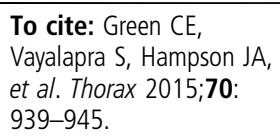

\section{ABSTRACT}

Introduction The PiSZ genotype results in less severe deficiency of alpha- 1 antitrypsin (AAT) than PiZZ. Less is known about phenotypic and prognostic features.

Methods We studied 699 PiZZ, 126 PiSZ and 316

PiMM patients. All AAT deficiency (AATD) patients were augmentation naive. PiSZ were compared with PiZZ patients for clinical phenotype at baseline including CT findings, smoke exposure, progression of lung disease and survival. Similarly, PiSZ patients diagnosed as a result of investigation for possible lung disease (lung index cases) were compared with PiMM. Multivariable analytical techniques and matching (PiSZ to PiZZ) were employed to account for demographic differences.

Results Pack-years smoked and FEV1 exhibited a negative correlation in PiSZ and ZZ patients (both $r=-0.43)$, with emphysema and COPD occurring more commonly in PiZZ patients at $<20$ pack-year exposure. In multivariable analyses, PiSZ patients were less likely to have emphysema $(p<0.01)$ and had better survival than PiZZ ( $p=0.017)$, but lung function decline did not differ significantly. $42 \%$ of PiSZ patients had upper-zonedominant emphysema on CT scan. Analyses of AAT level confirmed a critical threshold at $11 \mu \mathrm{M}$, particularly with regard to phenotypes classical of PiZZ AATD.

Significant baseline differences suggested that PiSZ had presented earlier to health services than PiMM. Once this was accounted for, risk of emphysema did not differ between PiSZ and PiMM although survival was lower in PiMM patients $(p<0.01)$.

Conclusions PiSZ patients are less susceptible to cigarette smoke than PiZZ. The pattern of emphysema may be similar at diagnosis to usual COPD.

\section{INTRODUCTION}

Alpha-1 antitrypsin deficiency (AATD) results from several deficiency alleles, with severe deficiency occurring in $\mathrm{Z}$ allele homozygotes (PiZZ), or carriers of null alleles leading to absence of $\mathrm{AAT}^{1}$ and is associated with early-onset emphysema. ${ }^{2}$ The $\mathrm{S}$ allele leads to a milder deficiency, and there has been considerable debate about whether heterozygous PiSZ patients are at increased risk of lung disease, largely due to the confounding factor of acquisition bias in some prior work. A meta-analysis concluded that PiSZ patients exhibit a risk of COPD over three times that of a normal individual; ${ }^{3}$ however, this result was influenced by one study showing a large increase in risk. ${ }^{4}$

\section{Key messages}

What is the key question?

- Are patients with the PiSZ genotype for alpha-1 antitrypsin deficiency (AATD) similar to classical PiZZ AATD or to usual COPD?

\section{What is the bottom line?}

- Many PiSZ patients look phenotypically similar to usual COPD at their first assessment; the interaction between their AAT level and smoke exposure appears critical in determining subsequent decline.

\section{Why read on?}

- Detailed phenotypic data, decline of lung function and mortality analyses are presented between PiSZ, PiZZ and PiMM, together with an exploration of threshold levels of smoke exposure in AATD and current knowledge pertinent to diagnosing and managing AATD.

Previous studies of PiSZ patients are summarised in online supplementary table 1.

Epidemiological studies suggest that there are $>500000$ PiSZ individuals in Europe, ${ }^{5}$ who typically exhibit an AAT level less than half that of a normal (PiMM) person. ${ }^{6}$ An interaction between AAT and cigarette smoke exposure occurs in murine models, where emphysema develops more rapidly. ${ }^{7}$ This, together with the available epidemiology, suggests that PiSZ patients should have a lung disease risk lying between PiMM and PiZZ patients, given the same level of smoke exposure. Consistent with this, we have shown in a relatively small cohort $(n=63)$, matched for smoking status, that PiSZ patients exhibit less severe lung disease than PiZZ. ${ }^{8}$ Even low levels of smoke exposure may be deleterious to lung function in PiZZ patients; however, an important threshold may occur at 20 pack-years, above which associations between FEV1 and pack-years are less apparent.' Whether this differs in PiSZ patients is unknown, and little is known about their prognosis. We sought to confirm the pattern of lung disease exhibited by PiSZ patients, explore the relationship between cumulative smoke exposure, AAT level and severity of disease, and compare prognosis between PiSZ, PiZZ and PiMM patients. 


\section{METHODS}

\section{Subjects}

The UK AATD registry (ADAPT) was established in 1996 and has been described in detail elsewhere. ${ }^{10}$ Briefly, patients undergo annual lung physiology, biological sample collection and clinical assessment. At enrolment most patients have a chest CT scan; quantitative CT analysis was undertaken by density mask analysis of $5 \mathrm{~mm}$ CT scan slices; voxel indices (VI) at -910 (the proportion of lung voxels below -910 Hounsfield Units $(\mathrm{HU}))$ and the ratio between the upper and lower zones calculated. ${ }^{11}$ Lung function decline was determined on all patients with $\geq 3$ years' follow-up; methods for calculation of decline have been described in our previous work. ${ }^{12}$ All patients with PiSZ and PiZZ genotypes were selected. Those diagnosed due to investigation for symptoms/signs of lung disease were termed lung index cases.

PiMM patients with usual COPD comprised all patients in the West Midlands COPD cohort (WMCC; described in our previous published work ${ }^{13}$ ) and all patients from the Chronic Diseases Resource Centre (CDRC; a University of Birmingham COPD patient registry, identified from local clinics). The CDRC has superceded the WMCC and has assessment procedures similar to the UK AATD registry. Data on CT density and decline in lung function were not available for PiMM patients, as referring centres used a variety of CT protocols, and had either not been followed up for sufficient time or had insufficient lung function data to calculate decline.

\section{Statistical analysis}

All analyses were carried out in SPSS (SPSS V.20; IBM, USA). Univariable analyses compared PiSZ and PiZZ patients for demographics, smoking status, index status, occupational exposure (assessment methods described in our previous work ${ }^{12}$ ), comorbidities, lung function, CT densitometry (where available) survival and lung function decline. Analyses were performed using $\chi^{2}$ tests for categorical variables and Mann-Whitney $U$ tests for continuous variables, substratifying by index status, smoking status and presence of COPD for analyses pertaining to decline. Co-variables for logistic, linear and Cox regression analyses were selected if $\mathrm{p}<0.1$ in univariable analysis and no significant collinearity with another included variable. Prior literature on influences on mortality ${ }^{14}$ and decline ${ }^{12} 15 \quad 16$ in AATD were used to aid prioritisation of co-variables where necessary. All results from the multivariable analysis are reported as two-tailed; significance was taken at $p<0.05$. Since there were demographic differences between PiSZ and PiZZ patients, for which subgroup analyses and statistical adjustment in regression analysis might be imperfect, an attempt at a matched analysis was also made (see online supplementary data). Within the whole AATD group, the relationship of AAT level to clinical phenotype and decline was conducted using similar techniques. Finally, univariable analyses were undertaken comparing lung index PiSZ and PiMM patients for demographics, smoking status, clinical phenotype and survival. Results guided logistic and linear regressions similar to the PiSZ versus PiZZ comparisons.

\section{RESULTS}

\section{Comparison of PiSZ and PiZZ patients}

Table 1 shows patients' characteristics and univariable analyses. PiZZ patients were more likely to be lung index cases and ex smokers, had higher rates of emphysema, bronchiectasis and chronic bronchitis, worse lung function (in all parameters), lower AAT levels and worse survival. Almost all (99.8\%) PiZZ patients and $11.3 \%$ PiSZ patients had AAT $<11 \mu \mathrm{M}$. CT scans were available on 37 non-index and 64 index PiSZ patients (index vs nonindex characteristics; see online supplementary table 2). Smoking appeared to be a greater influence on clinical phenotype than PiSZ versus PiZZ genotype (smoke exposed vs never smokers' characteristics; see online supplementary table 3 ).

Direct correlation between FEV1\% predicted and pack-years occurred up to a break point, which appeared similar to the published 20 pack-year threshold in PiZZ patients, though possibly higher in PiSZ (figure 1A, B). The proportion of emphysema and COPD cases occurring below 20 pack-years was greater in PiZZ patients (figure 1C, D). There was no difference in prevalence of common comorbidities (ischaemic heart disease (IHD), osteoporosis, anxiety, depression), age, pack-years smoked or occupational exposure risks (all $\mathrm{p}>0.2$ ). Multivariable logistic regressions, stratified for method of ascertainment of AATD, including smoke exposure and age as covariates, showed that emphysema was less common in lung index and family screened PiSZ patients (OR 0.15 and $0.03 ; \mathrm{p}=0.003$ and $<0.001$, respectively), while rates of chronic bronchitis and bronchiectasis were similar between groups (all $p>0.2$ ). In linear regressions stratified for method of ascertainment of AATD and adjusting for smoking status and age, lung index PiSZ patients had significantly better lung function compared with PiZZ (all parameters shown in table $1, \mathrm{p}<0.05$ ), while non-index PiSZ patients were better in most parameters (residual volume and total lung capacity no difference, other parameters $\mathrm{p}<0.05$ ). Matched analyses were no different (see online supplementary results). Differences in emphysema between PiSZ and PiZZ patients were more marked in the lower zones (figure 2); 42.9\% of PiSZ patients scanned had upper-zone-dominant emphysema compared with $14.1 \%$ of PiZZ (both $\mathrm{p}<0.01$ ).

Sufficient physiological data to calculate decline were available in $68 \mathrm{PiSZ}$ and 514 PiZZ patients, with a mean of five measures per PiZZ and four per PiSZ patient. $22.5 \%$ of PiZZ patients had COPD with FEV1 $<30 \%$ predicted $(\mathrm{n}=116), 33.5 \%$ had FEV1 30-50\% ( $n=172), 25 \%(n=129)$ had COPD with FEV1 $>50 \%$ predicted and the remainder did not have COPD at the start of the decline calculation period $(\mathrm{n}=97)$. In the PiSZ patients, these figures were $11.3 \%, 8.1 \%, 17.7 \%, 29 \%$ and $33.9 \%(\mathrm{n}=8,5,12,20$ and 23), respectively. Decline in FEV1 in some subgroups of interest is shown in table 2. Median decline was greatest when baseline FEV1 was $50-80 \%$ predicted (PiZZ $-56.3 \mathrm{~mL} / \mathrm{year}$, PiSZ $-65.3 \mathrm{~mL} / \mathrm{year}, \mathrm{p}=0.707$ ). There was no difference in annual decline of FEV1 or gas transfer (DLCO and KCO) \%predicted when analysed as categories (figure 3) or continuous variables. Regression analyses stratified for smoking status, presence of COPD and index status, adjusting for baseline lung function and age did not influence this similarity (all $\mathrm{p}>0.18$ for smoking status, $\mathrm{p}>0.33$ for COPD, $p>0.77$ for index status), nor did matching (see online supplementary results). Cox regression analyses, including age, smoking status, index status and baseline FEV1 as co-variables, showed significantly better survival in PiSZ patients (figure 4A).

We then explored the importance of AAT level with regard to susceptibility to smoke, subsequent development of lung disease and decline. When considered as a continuous variable, AAT level had no significant impact on logistic regressions seeking associations of emphysema, chronic bronchitis and bronchiectasis. However, a level of $\leq 11 \mu \mathrm{M}$ associated with development of emphysema $(\mathrm{p}<0.001)$, an upper zone $(\mathrm{UZ}) /$ lower zone (LZ) voxel index (VI) suggestive of lower-zone-dominant disease $(\mathrm{p}<0.001)$ and chronic bronchitis $(\mathrm{p}<0.001)$. There was a strong trend toward interaction between AAT $\leq 11 \mu \mathrm{M}$ and pack- 
Table 1 Clinical characteristics of patients

\begin{tabular}{|c|c|c|c|c|c|c|}
\hline \multirow[b]{2}{*}{ Feature } & \multirow{2}{*}{$\begin{array}{l}\text { PiZZ } \\
n=699\end{array}$} & \multirow{2}{*}{$\begin{array}{l}\text { PiSZ } \\
n=126\end{array}$} & \multirow{2}{*}{$\begin{array}{l}\text { PiSZ lung index cases } \\
\mathrm{N}=64\end{array}$} & \multirow{2}{*}{$\begin{array}{l}\text { PiMM } \\
\mathrm{N}=316\end{array}$} & \multicolumn{2}{|l|}{ p Value } \\
\hline & & & & & PiSZ vs PiZZ & PiSZ index vs PiMM \\
\hline Male & $404(57.8)$ & $71(56.3)$ & $38(59.4)$ & $182(57.7)$ & 0.717 & 0.810 \\
\hline Age & $50.8((16.4)$ & $53.5((20.0)$ & $55.4((1.5)$ & $68.4((13.6)$ & 0.021 & $<0.001$ \\
\hline Smoking status & & & & & $<0.001$ & 0.004 \\
\hline Ex smoker & $510(73.0)$ & $56(44.4)$ & $37(57.8)$ & $192(60.0)$ & & \\
\hline Never smoked & $161(23.0)$ & $43(34.1)$ & $15(23.4)$ & $3(0.9)$ & & \\
\hline Current smoker & $28(4.0)$ & $27(21.4)$ & $12(18.8)$ & $121(39.0)$ & & \\
\hline Pack-years smoked & $13.5((23.5)$ & 13.0 ((35.4) & $26.2((3.0)$ & 44.2 ((32.5) & 0.402 & $<0.001$ \\
\hline Reason for diagnosis & & & - & - & 0.007 & - \\
\hline Lung disease & $532(76.2)$ & $77(61.0)$ & & & & \\
\hline Family screening & $118(16.9)$ & $38(30.5)$ & & & & \\
\hline Liver disease & $11(1.6)$ & $1(0.8)$ & & & & \\
\hline Other reason & $38(5.4)$ & $10(7.9)$ & & & & \\
\hline COPD & $587(84.0)$ & $66(52.4)$ & $43(67.2)$ & $316(100)$ & $<0.001$ & $<0.001$ \\
\hline Emphysema & $528(75.5)$ & $46(36.5)$ & $35(54.7)$ & $257(81.3)$ & $<0.001$ & $<0.001$ \\
\hline Chronic bronchitis & $249(35.6)$ & $31(24.6)$ & $18(28.1)$ & $198(62.8)$ & 0.034 & 0.037 \\
\hline Bronchiectasis & $189(27.0)$ & $22(17.4)$ & $11(17.2)$ & $96(30.3)$ & 0.041 & $<0.001$ \\
\hline$A A T$ level & $4.0((2.1)$ & $14.4(4.4)$ & $14.9(3.7)$ & - & $<0.001$ & - \\
\hline FEV1pp & $47.9(45.6)$ & 94.5 (51.3) & $76.5(2.6)$ & $46.4(26.0)$ & $<0.001$ & $<0.001$ \\
\hline FEV1/FVC (\%) & $38.2(26.4)$ & 67.5 (37.5) & $55.0(2.7)$ & $43.3(20.0)$ & $<0.001$ & 0.004 \\
\hline$T L C p p$ & 115.3 (21.9) & 107.4 (18.3) & $109.2(1.9)$ & $103.0(20.2)$ & $<0.001$ & $<0.001$ \\
\hline$R V p p$ & 119.3 (54.9) & 96.9 (46.3) & $107.9(4.6)$ & 119.8 (53.8) & $<0.001$ & $<0.001$ \\
\hline DLCOpp & $69.74(36.0)$ & $86.3(34.2)$ & $76.5(3.1)$ & $48.6(23.9)$ & $<0.001$ & $<0.001$ \\
\hline KCOpp & $64.3(28.8)$ & $89.0(29.5)$ & $78.1(3.0)$ & $59.0(29.6)$ & $<0.001$ & $<0.001$ \\
\hline Follow-up time (years) & $10.4(5.3)$ & $7.0(8.5)$ & $7.8(0.6)$ & $3.2(3.0)$ & 0.021 & $<0.001$ \\
\hline Deceased & $172(24.6)$ & $10(7.9)$ & $7(10.9)$ & $91(28.8)$ & $<0.001$ & 0.003 \\
\hline
\end{tabular}

Data are shown as $\mathrm{n}(\%)$ or median (IQR). In PiSZ index cases, data are shown as mean (SE) due to normal distribution.

AAT, alpha-1 antitrypsin; DLCO, carbon monoxide diffusing capacity; KCO, carbon monoxide transfer coefficient; RV, residual volume; TLC, total lung capacity.
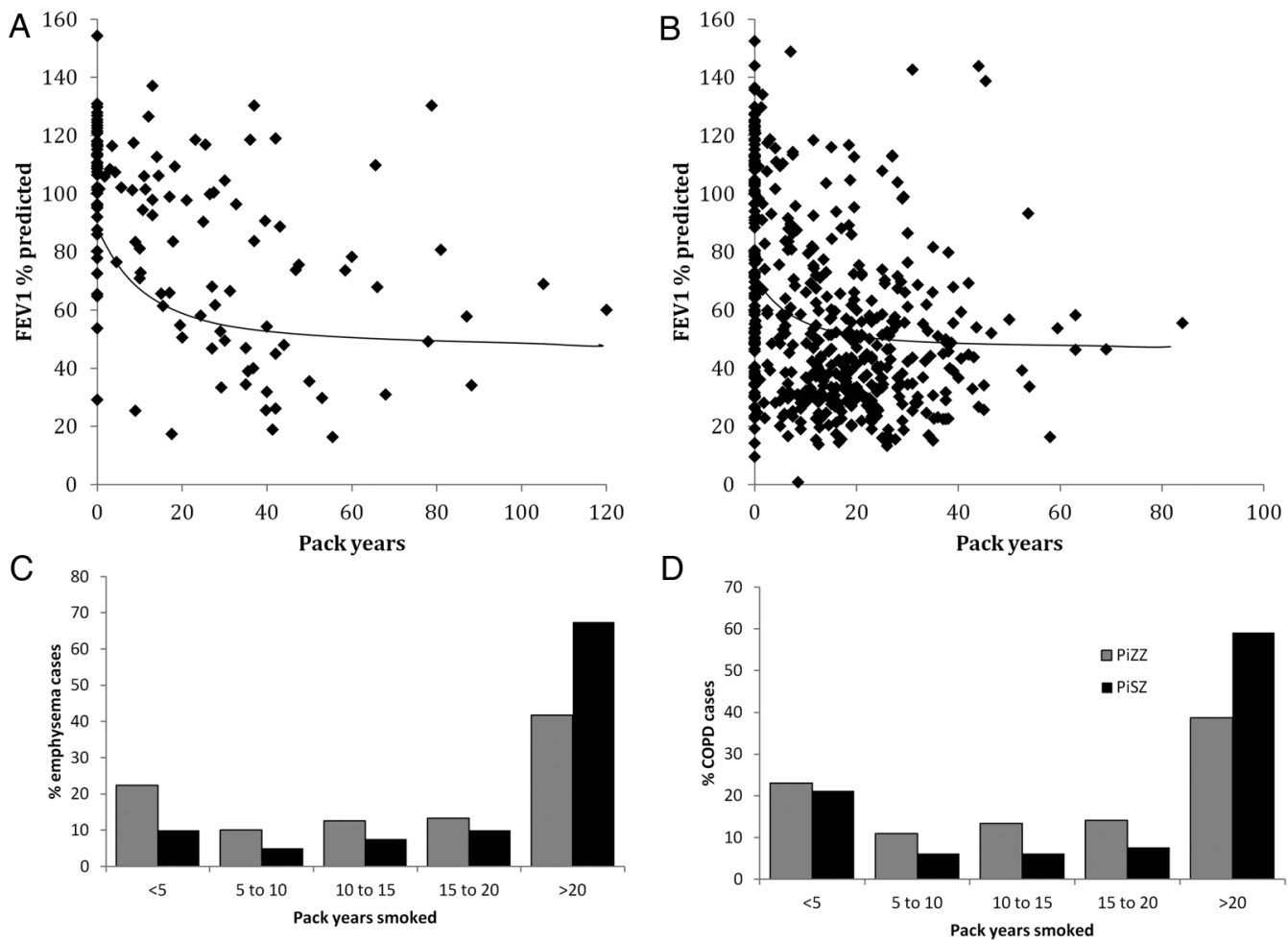

Figure 1 Relationship between pack-years smoked and lung function in alpha-1 antitrypsin deficiency. There was a linear relationship between baseline FEV1 and pack-years up to a threshold of 30 pack-years in PiSZ patients, which then disappeared $(A ; r=-0.43$ at $<30, r=-0.02$ at $\geq 30$ pack-years). The relationship was similar in PiZZ patients, but with a lower pack-year threshold ( $B ; r=-0.43$ at $<20, r=0.04$ at $\geq 20$ pack-years). Illustrative smoothed regression lines are shown on the graphs. Both emphysema (C) and COPD (D) were more common at exposures $<20$ pack-years in PiZZ patients. 

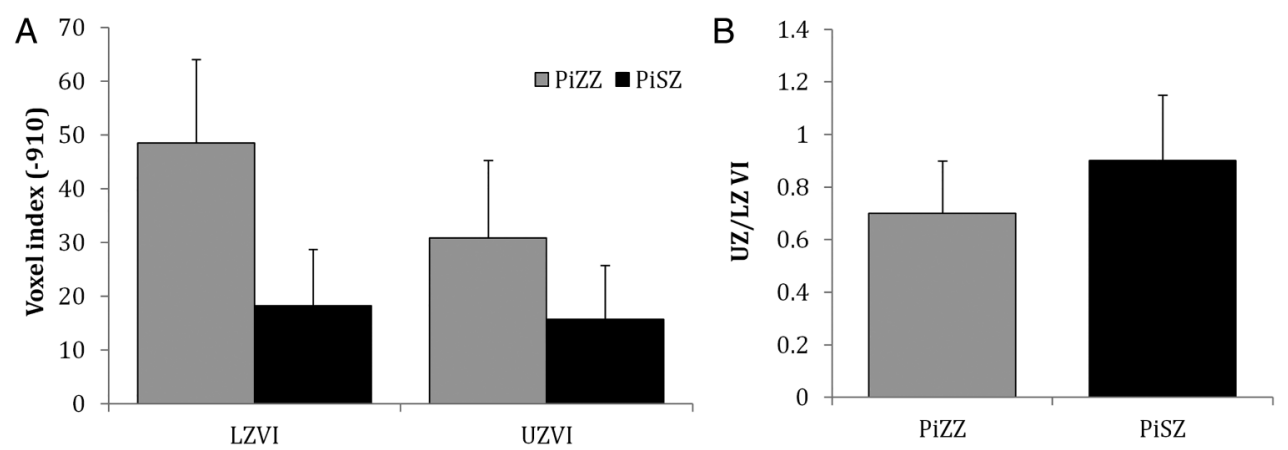

Figure 2 Characteristics of emphysema in alpha-1 antitrypsin deficiency patients. (A) CT density (VI-910) in upper and lower zones was worse in PiZZ with differences being marked in lower zone $(p<0.01)$ and marginal in upper zone. Voxel index $(\mathrm{VI})$ at -910 is the proportion of lung voxels below -910 Hounsfield Units (HU). (B) UZ/LZVI: Lower values demonstrate predominance of basal emphysema in PiZZ ( $p=0.001)$.

years smoked with regard to risk of chronic bronchitis $(p=0.09)$, but not other phenotypes. Furthermore, interaction between AAT level and pack-years smoked was significant in a multivariable general linear model for FEV1 decline (one-tailed test $\mathrm{p}=0.026$ ), whereas AAT level alone and PiSZ*pack-years interaction were not (both one-tailed $\mathrm{p}=0.150$ ).

\section{Comparison with usual COPD}

The primary reason for inclusion of PiMM patients was to see whether their phenotype was similar to PiSZ, hence univariable analyses were restricted to PiSZ index cases, as this eliminated ascertainment differences. Logistic regressions adjusting for age, smoke exposure and baseline FEV1 (different in univariable analyses; table 1) demonstrated that PiSZ patients had a similar risk of emphysema $(p=0.274)$ and bronchiectasis $(p=0.344)$, but lower risk of chronic bronchitis (OR 0.36 (0.18-0.72); $\mathrm{p}=0.016)$. Cox regression confirmed better survival in PiSZ patients (figure 4B).

\section{DISCUSSION}

We have confirmed that PiSZ patients have a lower risk of lung disease and are less susceptible to effects of cigarette smoke than PiZZ individuals, exhibiting a clinical phenotype, which is in many cases similar to usual PiMM COPD. Interpreting the prognostic data is more difficult due to issues of power and baseline differences between groups.

\section{Pulmonary phenotype}

This study is the largest to date on PiSZ AATD. In our earlier study, lung function and CT scan appearances concurred with those reported here; many PiSZ patients exhibited no emphysema, and many who did had upper-zone-dominant disease. ${ }^{8}$

Table 2 FEV1 decline (mL/year) in PiSZ and PiZZ alpha-1 antitrypsin deficiency

\begin{tabular}{llll}
\hline Variable & PizZ & PiSZ & p Value \\
\hline Current smoker & $-16.0(-8.1$ to -56.6$)$ & $-59.6(-16.8$ to -79.4$)$ & 0.287 \\
Ex smoker & $-37.0(-10.0$ to -76.1$)$ & $-41.8(-26.6$ to -88.5$)$ & 0.155 \\
Never smoker & $-41.6(-3.4$ to -77.7$)$ & $-33.6(-11.9$ to -66.9$)$ & 0.542 \\
COPD & $-38.2(-9.2$ to -76.1$)$ & $-52.3(-24.4$ to -94.3$)$ & 0.818 \\
No COPD & $-34.7(-7.5$ to -71.8$)$ & $-35.1(-13.6$ to -67.8$)$. & 0.120 \\
Lung index case & $-38.1(-8.6$ to -85.2$)$ & $-41.8(-19.7$ to -85.4$)$ & 0.278 \\
Non-lung index & $-38.1(-7.9$ to -82.4$)$ & $-36.5(-14.2$ to -67.7$)$ & 0.879 \\
case & & & \\
\hline
\end{tabular}

This agrees with data from an earlier, small, British PiSZ cohort ${ }^{17}$ and with much of the past PiSZ literature. The expected number of UK PiSZ patients exceeds PiZZ, ${ }^{5}$ yet there are far fewer PiSZ known to the registry. This may reflect ascertainment bias since AAT testing possibly occurs more frequently in patients with COPD exhibiting classical AATD lung disease (ie, lower-zonedominant emphysema). A missed diagnosis could have consequences since there is specific treatment for AATD lung disease in the form of AAT augmentation (available in the USA and many European countries, albeit not yet in the UK), which undiagnosed patients would not receive. Most international AATD guidelines specify that their scope is PiZZ patients or those with a circulating AAT level typical of PiZZ, and recommend the use of augmentation in the presence of emphysema and a specified level of FEV1. $^{18} 19$ This would exclude almost $90 \%$ of our PiSZ patients on the basis of AAT level. ${ }^{6}$ The main effect of augmentation is on progression of emphysema, best measured by CT densitometry, ${ }^{20}$ although effects on FEV1 decline have been reported in nonrandomised studies. ${ }^{21}$ Progression of emphysema on augmentation varies according to lung zone, ${ }^{11}$ hence augmentation might benefit PiSZ patients who exhibit lower-zone-dominant disease and low AAT levels (equivalent to PiZZ). Our data examining the threshold AAT level of $11 \mu \mathrm{M}$ support this, although specific studies in PiSZ patients would be required to determine this with confidence.

We also confirmed that PiSZ patients are less susceptible to smoke. A direct relationship between FEV1 and smoke exposure occurred below 30 pack-years in PiSZ and 20 pack-years in PiZZ patients. The reasons for this are discussed elsewhere, ${ }^{9}$ one of which is a 'floor effect', in which lung function has dropped sufficiently by the threshold that in many patients either no further decline occurs or they die. A smaller amount of 'resistant' smokers is also seen, adding to the lack of correlation at higher smoke exposures. While the data for the specific threshold were weak, the proportion of PiSZ patients developing emphysema or COPD at exposures $<20$ pack-years was also lower, supporting its existence. Moreover, the American NHLBI cohort reported the importance of smoking in disease development in PiSZ patients with AAT $<11 \mu \mathrm{M}$, alongside less lung function impairment than in PiZZ, thus supporting our data. ${ }^{22}$ A difference in the threshold at which smoke susceptibility occurs between PiSZ and ZZ patients is consistent with current understanding of AAT-neutrophil elastase (NE) interactions, whereby the area of obligate damage is exponentially related to AAT level, rising markedly at $<11 \mu \mathrm{M},{ }^{23}$ and therefore leading to a critical area of lung damage more quickly in PiZZ. Interestingly, among the PiSZ never smokers clinically significant 

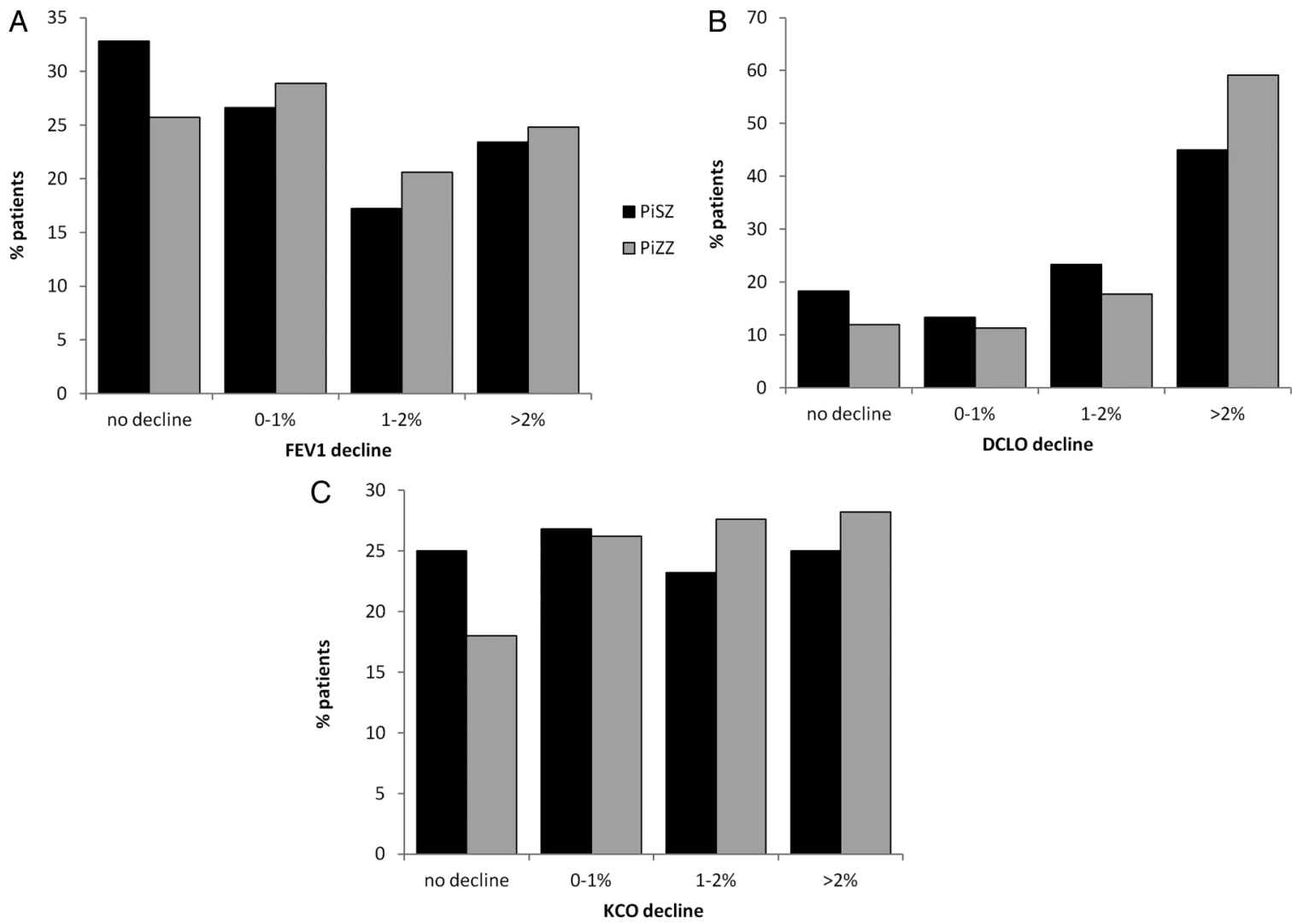

Figure 3 Decline in lung function in alpha-1 antitrypsin deficiency. The graphs show the proportion of patients with lung function that did not deteriorate, decreased by up to $1 \%$ of predicted (ie, fell from $50 \%$ predicted in year 1 to $\geq 49 \%$ predicted in year 2 ), between $1 \%$ and $2 \%$ predicted and $>2 \%$ predicted per year. (A) FEV1, (B) DLCO and (C) carbon monoxide transfer coefficient (KCO). There were no differences in the proportion of patients in each group (FEV1, $p=0.67 ; \mathrm{DLCO}, \mathrm{p}=0.22 ; \mathrm{KCO}=0.60$ ).

emphysema was uncommon, implying that in the absence of smoke it is not a risk factor-similar to PiMZ. ${ }^{24}$

We then compared PiSZ and MM patients to test our hypothesis that there would be a degree of phenotypic overlap, perhaps sufficient to make the two groups indistinguishable, thus accounting for missed diagnoses (relative to PiZZ). The potential impact of individuals with AATD diagnosed through family screening, who were not present in the usual COPD
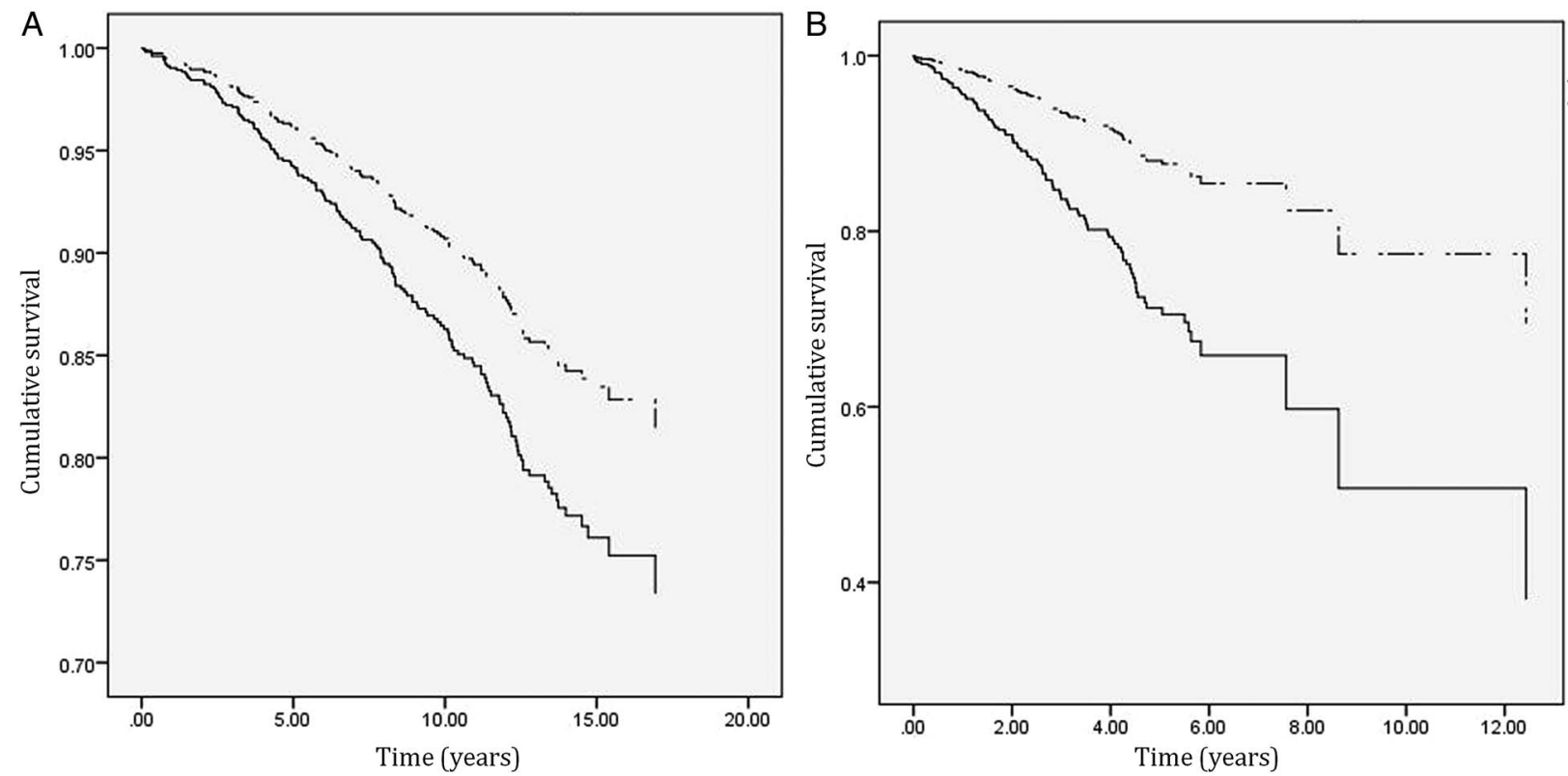

Figure 4 Cox regression demonstrating survival. (A) PiSZ (dashed line) versus PiZZ (solid line); analysis adjusted for baseline FEV1, age, index status and smoking. PiSZ patients had better survival ( $p=0.017$; log rank). (B) Lung index PiSZ (dashed line) versus PiMM (solid line); analysis adjusted for baseline FEV1, age and pack-years smoked. PiSZ patients had better survival $(p<0.001 ; \log$ rank). 
group, meant that statistical analyses compared lung index cases -risks of emphysema were similar, after adjustment for smoke exposure and age. This is consistent with PiSZ patients outside our cohorts being diagnosed less readily.

\section{Prognosis}

Our data implied that disease progression in PiSZ patients might be similar to PiZZ. This result was unexpected; PiSZ patients were generally better at baseline, which we have shown to relate to more rapid FEV1 decline, ${ }^{10} 16$ while PiZZ patients were worse, which we have shown to relate to more rapid gas transfer decline. ${ }^{1016}$ We therefore expected differences to occur in raw decline values, which would be less apparent after adjustment for baseline lung function and smoke exposure. However, raw values were similar and remained so regardless of the method by which we attempted to control for baseline differences. This may be explicable if AAT level is more critical than PiSZ genotype; consistent with this our data implied that AAT level, rather than PiSZ genotype, interacted with smoke exposure in the lung function decline models. AAT level does vary among PiSZ patients, presumably due to modifying effects of other genes or environmental factors, which is one reason why current guidelines for treatment take this feature into account.

No prior PiSZ studies have reported FEV1 decline (see online supplementary table 1); however, there are data available on decline in PiZZ, where loss of FEV1 was generally $60-80 \mathrm{~mL} /$ year. $^{15}$ 25-28 This is somewhat higher than the median in our PiZZ patients. Notably, the largest prior PiZZ study ${ }^{25}$ (see online supplementary table 4) exhibited both starting FEV1 (49\% predicted) and FEV1 decline similar to many of our PiZZ patients $(54 \mathrm{~mL} /$ year vs $56.3 \mathrm{~mL} /$ year in our FEV1 $50-80 \%$ subgroup). Our lower median is explicable by cohort characteristics. There are two groups in whom FEV1 decline is known to be lower-those with FEV1 $<30 \%$ predicted $^{10}$ or with no emphysema on their CT, who comprise $22.5 \%$ and $26.3 \%$ of our AATD cohort, respectively. High rates of patients with FEV1 $<30 \%$ account for the low decline seen in our PiZZ current smokers, whose minimum FEV1 was just 12\%, thus had no lung function to lose. Furthermore, our method of determining decline may be more accurate compared with previous studies, which calculated decline from just two data points, ${ }^{25}$ or over only 2 years in some patients. ${ }^{28}$ Thus we feel our decline data are accurate and comparable to other published data, once our cohorts' characteristics are taken into account. However, we acknowledge that a survivor bias, and relatively small numbers, in the decline analyses may have limited power to detect true differences between PiSZ and ZZ patients. While we tried to adjust for baseline differences in lung function (eg,11.3\% of patients had FEV $1<30 \%$ predicted in the PiSZ decline group compared with $22.5 \%$ of the PiZZ), statistical adjustment could have been incapable of fully accounting for differences. To overcome this, we attempted matching (see online supplementary data), which again showed no difference in decline. However, high smoke exposures in some PiSZ made matching imperfect also. Further studies of decline in PiSZ patients are therefore indicated.

Despite apparently similar lung function decline, subsequent survival was better in PiSZ compared with PiZZ. There are several possible reasons for these apparently inconsistent results. First, declining lung function may not be a good predictor of survival; we have recently shown that declining CT densitometry may be a better measure. ${ }^{29}$ Alternatively, it may be that neither statistical adjustments nor matching were capable of fully compensating for baseline differences, such that better survival reflects their higher starting point. One factor unlikely to have influenced the result is comorbidity, which did not differ between PiSZ and ZZ patients. Survival was worse in usual COPD than PiSZ patients, probably due to higher comorbidity burden attendant on greater smoke exposure, although we had insufficient data to test this hypothesis. The proportion of patients surviving 5 years was about $70 \%$ in usual COPD and between $90 \%$ and $95 \%$ in both PiSZ and ZZ individuals (figure 4). Since our AATD cohort is a national centre, and many patients travel some distance to be seen, there may be a survivor bias, which could have inflated the difference seen between PiSZ and MM. If present, it would have affected PiSZ and $\mathrm{ZZ}$ patients equally, hence their comparative survival result is more robust.

\section{Strengths and limitations}

The main strengths of our study are the large number of AATD patients, depth of clinical phenotyping and length of follow-up. Ideally we would have compared emphysema zone and lung function decline between PiSZ and MM, as we did for PiSZ versus ZZ, but were limited by lack of data. However, its utility for clinical management would be small since optimal management of COPD should occur in both usual COPD and COPD due to PiSZ AATD. The main difference would emerge if augmentation were available, for which the more informative longitudinal PiSZ comparison is with PiZZ patients. Further work to clarify whether differences in neutrophil function occur between PiSZ and MM patients, as they do between PiZZ and $\mathrm{MM}^{30}$ whether NE activity differs ${ }^{31} 32$ between groups and the utility of augmentation in PiSZ patients may be valuable follow-up studies.

Acknowledgements The authors would like to thank all staff at the UK AATD registry, particularly Di Griffiths and Ross Edgar, for their help in data collection, and all staff at Queen Elizabeth and Heartlands Hospitals who helped with data collection for the WMCC and CDRC cohorts.

Contributors CEG, SV, DM and JAH collected data. CEG, SV and AMT analysed data. CEG and AMT drafted the manuscript. RAS and AMT reviewed the manuscript. AMT acts as guarantor.

Funding AMT has current funding from the Alpha 1 Foundation, Linde REAL fund, MRC and EPSRC. CG is funded by the West Midlands Chest Fund.

Competing interests The UK AATD registry has received unrestricted and/or investigator initiated project funds within the last five years from two manufacturers of AAT augmentation therapy: Grifols Biotherapeutics and CSL Behring.

Patient consent Obtained.

Ethics approval South Bham (AATD and WMCC), North West (CDRC).

Provenance and peer review Not commissioned; externally peer reviewed.

\section{REFERENCES}

1 Salahuddin P. Genetic variants of alpha1-antitrypsin. Curr Protein Pept Sci 2010;11:101-17.

2 Laurell CB, Eriksson $\mathrm{S}$. The electrophoretic alpha 1 globulin pattern of serum in alpha 1 antitrypsin deficiency. Scand J Clin Lab Invest 1963;15:132-40.

3 Dahl M, Hersh CP, Ly NP, et al. The protease inhibitor PI*S allele and COPD: a meta-analysis. Eur Respir J 2005;26:67-76.

4 Bartmann K, Fooke-Achterrath M, Koch G, et al. Heterozygosity in the Pi-system as a pathogenetic cofactor in chronic obstructive pulmonary disease (COPD). Eur J Respir Dis 1985;66:284-96.

5 Blanco I, de Serres FJ, Fernandez-Bustillo E, et al. Estimated numbers and prevalence of $\mathrm{PI}^{*} \mathrm{~S}$ and $\mathrm{PI}^{*} \mathrm{Z}$ alleles of alpha1-antitrypsin deficiency in European countries. Eur Respir J 2006;27:77-84.

6 Ferrarotti I, Thun GA, Zorzetto $M$, et al. Serum levels and genotype distribution of alpha1-antitrypsin in the general population. Thorax 2012;67:669-74.

7 Takubo Y, Guerassimov A, Ghezzo H, et al. Alpha1-antitrypsin determines the pattern of emphysema and function in tobacco smoke-exposed mice: parallels with human disease. Am J Respir Crit Care Med 2002:166(12 Pt 1):1596-603.

8 Holme J, Stockley RA. CT scan appearance, densitometry, and health status in protease inhibitor SZ alpha1-antitrypsin deficiency. Chest 2009;136:1284-90. 
9 Castaldi PJ, DeMeo D, Kent DM, et al. Development of predictive models for airflow obstruction in alpha 1 antitrypsin deficiency. Am J Epidemiol 2009;170:1005-13.

10 Pillai AP, Turner AM, Stockley RA. Relationship of the 2011 Global Initiative for Chronic Obstructive Lung Disease strategy to clinically relevant outcomes in individuals with alpha1-antitrypsin deficiency. Ann Am Thorac Soc 2014;11:859-64.

11 Parr DG, Sevenoaks M, Deng C, et al. Detection of emphysema progression in alpha 1-antitrypsin deficiency using CT densitometry; methodological advances. Respir Res 2008;9:21

12 Wood AM, Harrison RM, Semple $S$, et al. Outdoor air pollution is associated with rapid decline of lung function in alpha-1-antitrypsin deficiency. Occup Environ Med 2010;67:556-61.

13 Wood AM, de Pablo P, Buckley CD, et al. Smoke exposure as a determinant of auto-antibody titre in AATD and COPD. Eur Respir J 2011;37:32-8.

14 Dawkins $P$, Wood A, Nightingale $P$, et al. Mortality in alpha-1-antitrypsin deficiency in the United Kingdom. Respir Med 2009;103:1540-7.

15 Seersholm N, Kok-Jensen A, Dirksen A. Decline in FEV1 among patients with severe hereditary alpha 1-antitrypsin deficiency type PiZ. Am J Respir Crit Care Med 1995; 152(6 Pt 1):1922-5

16 Dawkins PA, Dawkins CL, Wood AM, et al. Rate of progression of lung function impairment in alpha1-antitrypsin deficiency. Eur Respir J 2009;33:1338-44.

17 Hutchison DC, Tobin MJ, Cook PJ. Alpha 1 antitrypsin deficiency: clinical and physiological features in heterozygotes of Pi type SZ. A survey by the British Thoracic Association. Br J Dis Chest 1983;77:28-34.

18 American Thoracic Society, European Respiratory Society. American Thoracic Society/ European Respiratory Society statement: standards for the diagnosis and management of individuals with alpha-1 antitrypsin deficiency. Am J Respir Crit Care Med 2003;168:818-900.

19 Marciniuk DD, Hernandez P, Balter M, et al. Alpha-1 antitrypsin deficiency targeted testing and augmentation therapy: a Canadian Thoracic Society clinical practice guideline. Can Respir J 2012;19:109-16.

20 Dirksen A, Piitulainen E, Parr DG, et al. Exploring the role of CT densitometry: a randomised study of augmentation therapy in alpha1-antitrypsin deficiency. Eur Respir J 2009;33:1345-53.
21 Chapman KR, Stockley RA, Dawkins C, et al. Augmentation therapy for alpha1 antitrypsin deficiency: a meta-analysis. COPD 2009;6:177-84.

22 Turino GM, Barker AF, Brantly ML, et al. Clinical features of individuals with $\mathrm{PI}^{*} \mathrm{SZ}$ phenotype of alpha 1-antitrypsin deficiency. alpha 1-Antitrypsin Deficiency Registry Study Group. Am J Respir Crit Care Med 1996;154(6 Pt 1):1718-25.

23 Campbell EJ, Campbell MA, Boukedes SS, et al. Quantum proteolysis by neutrophils: implications for pulmonary emphysema in alpha 1-antitrypsin deficiency. J Clin Invest 1999;104:337-44.

24 Molloy K, Hersh CP, Morris VB, et al. Clarification of the risk of chronic obstructive pulmonary disease in alpha1-antitrypsin deficiency PiMZ heterozygotes. Am J Respir Crit Care Med 2014;189:419-27.

25 Survival and FEV1 decline in individuals with severe deficiency of alpha1-antitrypsin. The Alpha-1-Antitrypsin Deficiency Registry Study Group. Am J Respir Crit Care Med 1998;158:49-59.

26 Hutchison DC, Cooper D. Alpha-1-antitrypsin deficiency: smoking, decline in lung function and implications for therapeutic trials. Respir Med 2002;96:872-80.

27 Wu MC, Eriksson S. Lung function, smoking and survival in severe alpha 1-antitrypsin deficiency, PizZ. J Clin Epidemiol 1988:41:1157-65.

28 Burdon JG, Brenton S, Hocking V, et al. Decline in FEV1 in patients with PiZ alpha-1-antitrypsin deficiency: the Australian experience. Respirology 2002;7:51-5

29 Green CE, Turner AM. Lung density decline predicts survival and quality of life outcomes in patients with Alpha 1 antitrypsin deficiency. Thorax 2014;69(Suppl 2):A12.

30 Sapey E, Wood AM, Ahmad A, et al. Tumor necrosis factor-\{alpha\} rs361525 polymorphism is associated with increased local production and downstream inflammation in chronic obstructive pulmonary disease. Am J Respir Crit Care Med 2010;182:192-9.

31 Carter RI, Mumford RA, Treonze KM, et al. The fibrinogen cleavage product Aalpha-Val360, a specific marker of neutrophil elastase activity in vivo. Thorax 2011;66:686-91.

32 Carter RI, Ungurs MJ, Mumford RA, et al. Aalpha-Val360: a marker of neutrophil elastase and COPD disease activity. Eur Respir J 2013;41:31-8. 